\title{
Performance and Analysis of Reactive Power Compensation by Unified Power Flow Controller
}

\author{
Yogita Kumari ${ }^{* 1}$, Anmol Gupta ${ }^{2}$, Shiv Prakash Bihari ${ }^{3}$, R.N.Chaubey ${ }^{4}$, B. Sehgal ${ }^{5}$ \\ ${ }_{1,2}$ Krishna Institute of Engineering and Technology (KIET), Ghaziabad, India \\ 3,4,5 Inderprastha Engineering College (IPEC), Ghaziabad, India \\ *Corresponding author, e-mail: yogitaaryanipec@gmail.com ${ }^{1}$, spbiharinit@gmail.com ${ }^{3}$
}

\begin{abstract}
The Unified Power Flow Controller (UPFC) is the most versatile of the FACTS controllers envisaged so far. The main function of the UPFC is to control the flow of real and reactive power by injection of a voltage in series with the transmission line. Both the magnitude and the phase angle of the voltage can be varied independently. Real and Reactive power flow control can allow for power flow in prescribed routes, loading of transmission lines close to their thermal limits and can be utilized for improving transient and small signal stability of the power system. In this paper UPFC is incorporated in a SMIB (Single Machine Infinite Bus) system and the response of SMIB system has been recorded with and without UPFC, thereafter the comparison of both the output has been done. When no UPFC is installed, real and reactive power through the transmission line cannot be controlled. This paper presents control and performance of UPFC intended for installation on that transmission line to control power flow. Installing the UPFC makes it possible to control amount of active power flowing through the line. Simulations are carried out using MATLAB software to validate the performance of the UPFC.
\end{abstract}

Keywords: single machine infinite bus bar, thyristor controlled reactor, UPFC, Series convertor, fixed capacitor, fuzzy controller, reactive power

\section{Introduction}

The steady-state security of power system has become a matter of grave concern in system operation and planning [1-4]. As the power system becomes more complex and more heavily loaded, it cannot be operated in unstable or insecure situations like the cascading thermal overloads, the frequency collapse, and the voltage instability. Moreover, as electric power transaction increases in the deregulated environment, it incurs a serious impact on the system's security [5].

Power system security means the ability to withstand a set of credible contingencies caused by internal or external factors concerning power system and to continue operating without interrupting power supply that is supplied to the customers [6]. For a secure operation of the power system, it is important to maintain the required level of security margin. Problems concerning security of a system can be controlled at any part of the power system operation, i.e. generation, transfer and loads [7]. In the generation part, it can increase a power system's security and support its low voltage bus by re-dispatching generator outputs. The security of the power system can also be ensured by demand-side management with respect to interruptible loads [8].

The need for more efficient and fast responding electrical systems has given rise to innovative technologies in transmission using solid-state devices. These are called FACTS devices which enhance stability and increase line loadings closer to thermal limits. The development of power semiconductor devices with turn-off capability (GTO, MCT) opens up new perspectives in the development of FACTS devices [9]. FACTS devices are the key to produce electrical energy economically and environmental friendly in future [10].

Flexible alternating current transmission systems (FACTS) technology opens up new opportunities for controlling power and enhancing the usable capacity of present, as well as new and upgraded lines [3]. The Unified Power Flow Controller (UPFC) is a second generation FACTS device, which enables independent control of active and reactive power besides improving reliability and quality of the supply [4]. 


\section{Single Machine Infinite Bus System} shown as:

First task is to develop the simulink model for the SMIB system. A SMIB system can be

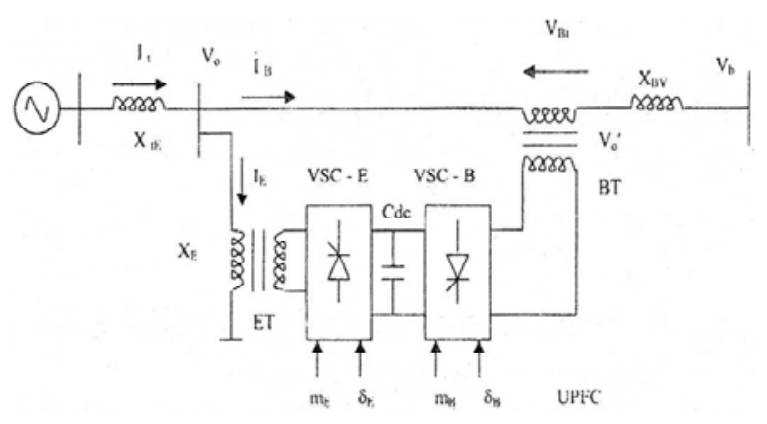

Figure 1. Single machine Infinite bus system

The above Figure 1 shows a SMIB system along with a UPFC. Here a single machine, i.e. a generator is connected to it as a source machine for which the deviations in speed and phase angle will be studied and modeled later.

\section{Unified Power Flow Controller (UPFC)}

The UPFC is the most versatile and complex power electronic equipment that has emerged for the control and optimization of power flow in electrical power transmission systems. It offers major potential advantages for the static and dynamic operation of transmission lines.

The UPFC was devised for the real-time control and dynamic compensation of ac transmission systems, providing multifunctional flexibility required to solve many of the problems facing the power industry. Within the framework of traditional power transmission concepts, the UPFC is able to control, simultaneously or selectively, all the parameters affecting power flow in the transmission line. Alternatively, it can independently control both the real and reactive power flow in the line unlike all other controllers.

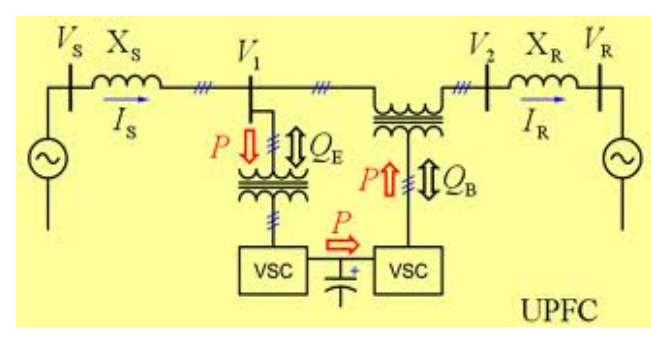

Figure 2. Diagram of UPFC

Unified power flow controller (UPFC) is a combination of static synchronous compensator (STATCOM) and a static synchronous series compensator (SSSC) which are coupled via a common dc link, to allow bi-directional flow of real power between the series output terminals of the SSSC and the shunt output terminals of the STATCOM and are controlled to provide current real and reactive series line compensation without an external electric energy source. The UPFC, by means of angularly unconstrained series voltage injection, is able to control, concurrently or selectively, the transmission line voltage, impedance and angle or alternatively, the real and reactive power flow in the line. The UPFC may also provide independently controllable shunt reactive compensation. Viewing the operation of the UPFC from the standpoint of conventional power transmission based on reactive shunt compensation, series compensation and phase shifting, the UPFC can fulfil all these functions

IJEEI Vol. 3, No. 3, September 2015: $141-149$ 
and thereby meet multiple control objectives by adding the injected voltage $V_{B t}$ with appropriate amplitude and phase angle, to the terminal voltage $\mathrm{V} O$.

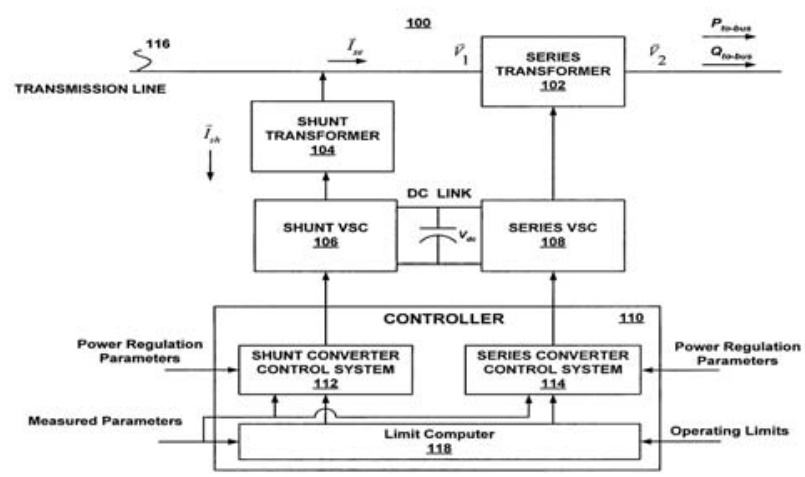

Figure 3. Block diagram of UPFC

\subsection{Basic Principle of UPFC}

The UPFC consists of two voltage sourced converters, connected back-to-back and are operated from a common dc link provided by a storage capacitor. The arrangement functions as an ideal ac-to-ac power converter in which the real power can freely flow in either direction between the ac terminals of the two converters, and each converter can independently generate(or absorb) reactive power at its own ac output terminal. Converter 2 provides the main function of the UPFC by injecting a voltage $\mathbf{V}_{\mathrm{pq}}$ with controllable magnitude $V_{p q}$ and phase angle $\rho$ in series with the line via an insertion transformer. This injected voltage acts essentially as asynchronous ac voltage source. The transmission line current flows through this voltage source resulting in reactive and real power exchange between it and the ac system. The reactive power exchanged at the ac terminal is generated internally by the converter. The real power exchanged at the ac terminal is converted into dc power which appears at the dc link as a positive or negative real power demand. The basic function of converter 1 is to supply or absorb the real power demanded by converter 2 at the common dc link to support the real power exchange resulting from the series voltage injection. This dc link power demand of converter2 is converted back to ac by converter 1 and coupled to the transmission line bus via a shunt connected transformer. In addition to the real power need of converter 2, converter 1 can also generate or absorb controllable reactive e power, if it is desired, and thereby provide independent shunt reactive compensation for the line. It is important to note that whereas there is a closed direct path for the real power negotiated by the action of reactive power exchanged is supplied or absorbed locally by converter 2 and therefore does not have to be transmitted by the line. Thus, converter 1 can be operated at a unity power factor or be controlled to have a reactive power exchange with the line independent of the reactive power exchanged by converter 2. Obviously, there can be no reactive power flow through the UPFC dc link.

\subsection{Control Structure of UPFC}

The UPFC control system is divided into internal (or converter) control and functional operation control. The internal controls operate the two converters so as to produce the commanded series injected voltage and, simultaneously, draw the desired shunt reactive current. The internal controls provide gating signals to the converter valves so that the converter output voltages will properly respond to the internal reference variables, in accordance with the basic control structure. As it can be observed, the series converter responds directly and independently to the demand for series voltage vector injection. Changes in series voltage vector, $V_{p q}$ can therefore be affected virtually instantaneously. In contrast, the shunt converter operates under a closed-loop current control structure whereby the shunt real and reactive power components are independently controlled. The shunt reactive power responds directly to an input demand. However, the shunt real power is dictated by another control loop that acts to maintain a preset voltage level on the dc link, thereby providing the real power supply or sink for 
the real power Ensures the required real power balance between the two converters. The converters do not exchange reactive power through the link. The external or functional operation control defines the functional operating mode of the UPFC and is responsible for generating the internal references, $V_{\text {pqRef }}$ and $I_{\text {pqref }}$ for the series and shunt compensation to meet the prevailing demands of the transmission system. The functional Operating modes and compensation demands, represented by external (or system) reference inputs, can be set manually by the operator or dictated by an automatic system optimization control to meet specific operating and contingency requirements. The capability of unrestricted series voltage injection together with independently controllable reactive power exchange offered by the circuit structure of two back-to back converters, facilitate several operating and control modes for the UPFC. These include the option of reactive shunt compensation and the free control of series voltage injection according to a prescribed functional approach selected for power flow control. The UPFC circuit structure also allows the total decoupling of the two converters to provide independent reactive shunt compensation (STATCOM) and reactive series compensation (SSSC) without any real power exchange.

\section{Basic Operating Modes of UPFC \\ 4.1. Shunt Converter}

The shunt converter is operated so as to draw a controlled current, $I_{\text {shq }}$ from the line. One component of this current, $\mathrm{I}_{\text {shp }}$ is automatically determined by the requirement to balance the real power of the series converter. The other component, $I_{\text {shq }}$, is reactive and can be set to any desired reference level (inductive or capacitive) within the capability of the converter. The reactive compensation control modes of the shunt converter are, of course, very similar to those commonly employed for the STATCOM and conventional static var compensator.

\subsubsection{Reactive Power Control Mode}

In reactive power control mode the reference input is an inductive or capacitive var request. The shunt converter control translates the var reference into a corresponding shunt current request and adjusts the gating of the converter to establish the desired current.

\subsubsection{Automatic Voltage Control Mode}

In voltage control mode, the shunt converter reactive current is automatically regulated to maintain the transmission line voltage to a reference value at the point of connection, with a defined droop characteristic. The droop factor defines the per unit voltage error per unit of Converter reactive current within the current range of the converter. The automatic voltage control uses voltage feedback signals, usually representing the magnitude of the positive sequence component of bus voltage, $\mathrm{v}_{1}$.

\subsection{Series Converter}

The series converter controls the magnitude and angle of the voltage vector $V_{p q}$ injected in series with the line. This voltage injection is, directly or indirectly, always intended to influence the flow of power on the line. However, $V_{p q}$ is dependent on the operating mode selected for the UPFC to control power flow. The principal operating modes are as follows.

\subsubsection{Direct Voltage Injection Mode}

The series converter simply generates the voltage vector, $\mathrm{V}_{\mathrm{pq}}$ With the magnitude and phase angle requested by the reference input. This operating mode may be advantageous when a separate system optimization control coordinates the operation of the UPFC and other FACTS controllers employed in the transmission system.

\subsubsection{Bus Voltage Regulation and Control Mode}

The injected voltage vector, $\mathrm{V}_{\mathrm{pq}}$, is kept in phase with the "input" bus voltage vector $\mathrm{v}_{1}$ and its magnitude is controlled to maintain the magnitude "output" bus voltage vector $v_{2}$ at the given reference value. 


\subsubsection{Line Impedance Compensation Mode}

The magnitude of the injected voltage vector, $V_{p q}$, is controlled in proportion to the magnitude of the line current, so that the series insertion emulates impedance when viewed from the line. The desired impedance is specified by reference input and in general it may be complex impedance with resistive and reactive components of either polarity. A special case of impedance compensation occurs when the injected voltage is kept in quadrature with respect to line current to emulate purely reactive (capacitive or inductive) compensation. This operating mode may be selected to match existing series capacitive line compensation in the system.

\subsubsection{Phase Angle Regulation Mode}

The injected voltage vector $\tilde{v}_{p q}$ is controlled with respect to the "input" bus voltage vector $\tilde{\mathbf{v}}_{1}$ so that the "output" bus voltage vector $\tilde{\mathbf{v}}_{2}$ is phase shifted, without any magnitude change, relative to $\tilde{\mathbf{v}}_{1}$ by an angle specified by the reference input. One special case of phase shifting occurs when $\tilde{\mathbf{v}}_{\mathrm{pq}}$ is kept in quadrature with $\tilde{\mathbf{v}}_{1}$ to emulate the "quadrature booster."

\subsubsection{Automatic power flow control mode}

The magnitude and phase angle of the injected voltage vector, $\tilde{\mathbf{v}}_{\mathrm{pq}}$, is controlled so as to force such a line current vector, $\tilde{\mathbf{i}}$, that results in the desired real and reactive power flow in the line. In automatic power flow control mode, the series injected voltage is determined automatically and continuously by a closed -loop control system to ensure that the desired $\mathrm{P}$ and $Q$ are maintained despite power system changes. The transmission line containing the UPFC thus appears to the rest of the power system as a high impedance power source or sink. This operating mode, which is not achievable with conventional line compensating equipment, has far reaching possibilities for power flow scheduling and management. It can also be applied effectively to handle dynamic system disturbances (eg. to damp power oscillations).

\section{Fuzzy Logic Approach}

Fuzzy logic is an innovative technology that enhances conventional system design with engineering expertise. The use of fuzzy logic can help to circumvent the need for rigorous mathematical modelling. Fuzzy logic is a true extension of conventional logic, and fuzzy logic controllers are a true extension of linear control models. Hence anything that was built using conventional design techniques can be built with fuzzy logic, and vice-versa. However, in a number of cases, conventional design methods would have been overly complex and, in many cases, might prove simpler, faster and more efficient. The key to successful use of fuzzy logic is clever combination with conventional techniques. Also, a fuzzy system is time-invariant and deterministic. Therefore any verification and stability analysis method can be used with fuzzy logic, tool. For a upfc, a fuzzy logic approach is applied to coordinate three control inputs: inphase voltage control; quadrature voltage control; and shunt compensation, of a unified power flow controller to improve the transient stability of a power system. The fuzzy rules for the controller are created based on a bang-bang control strategy whose objectives are minimising the first swing, damping the oscillations at an optimum rate and maximising the transient stability margin. The proposed controller also incorporates a technique of reducing the chattering of generator output which naturally results from the application of bang-bang control. Simulation results show that the fuzzy logic based controller provides satisfactory performance, meeting the design objectives. The results also show the robustness of the controller. The designed fuzzy-based UPFC controller adjusts four UPFC inputs by appropriately processing of the input error signal, and provides an efficient damping. The results of the simulation show that the UPFC with fuzzy-based controllers is more effective in damping Low Frequency Oscillations compared to UPFC with PID controllers. 


\section{Simulink Model of UPFC} installed in it.

The above Figure 4 shows, the simulink model of a SMIB system without UPFC

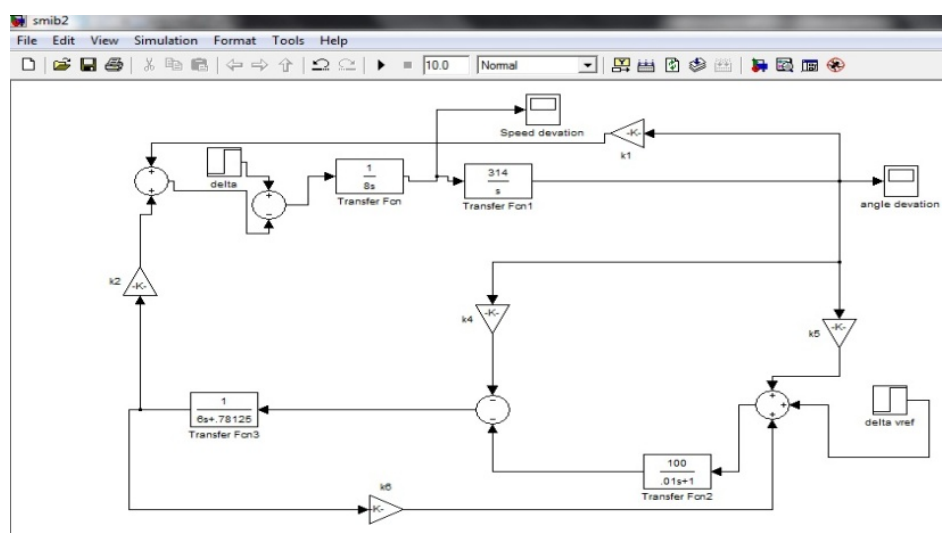

Figure 4. Simulink model of SMIB without UPFC

\subsection{Output of the SMIB System without UPFC}

Figure 5 and Figure 6 show that the output of a SMIB system without any damping is of oscillatory nature and is not stabilized as it should be.

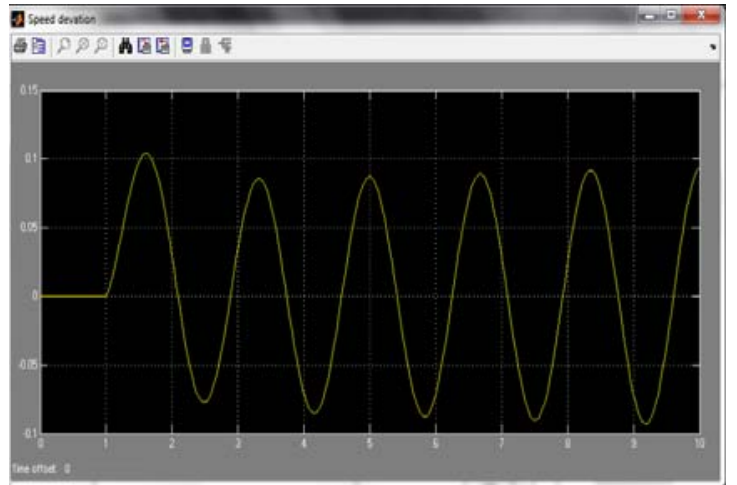

Figure 5. Speed deviation without UPFC

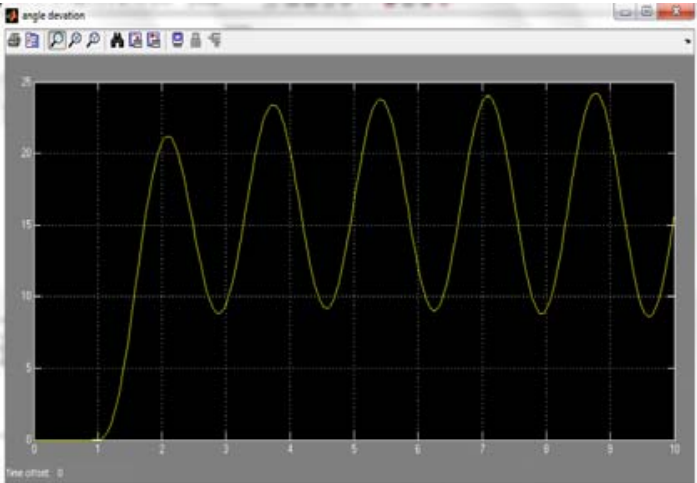

Figure 6. Phase angle deviation Without UPFC

\section{SMIB System with UPFC}

After the implementation of SMIB system which is under investigation, now the system installed with UPFC is implemented and studied.The following model is the basis of simulink model of UPFC damping controller,

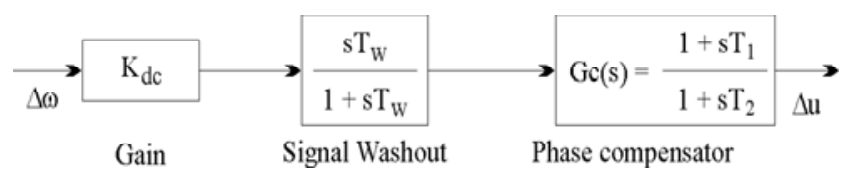

Figure 7. Simulink model of UPFC damping controller 
Here, the value of natural frequency is computed and then multiplied by the specified gain constant which is then passed to the signal washout block which is nothing but a high pass filter. This high pass filter prevents steady changes in the speed from modifying the UPFC input parameter. Figure 8 shows the simulink model of SMIB system installed with an UPFC damping controller and Figure 9 is the simulink model of the damping controller.

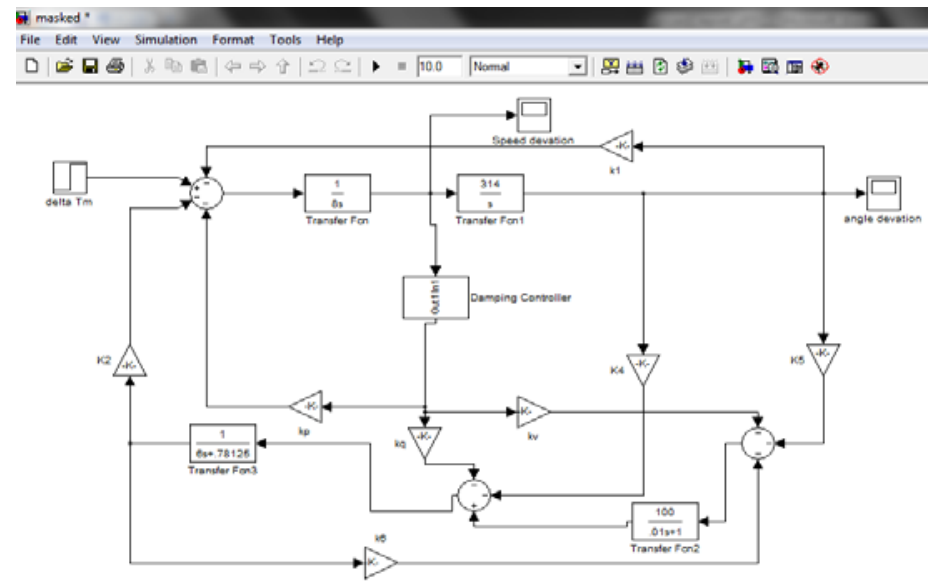

Figure 8. Simulink model with UPFC

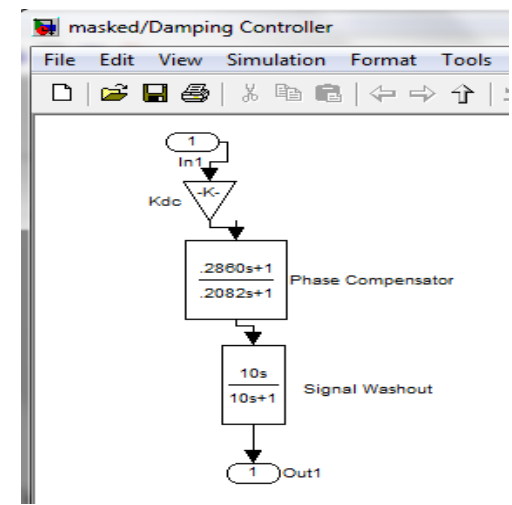

Figure 9. Simulink With External Devices

\subsection{Output of SMIB System with UPFC}

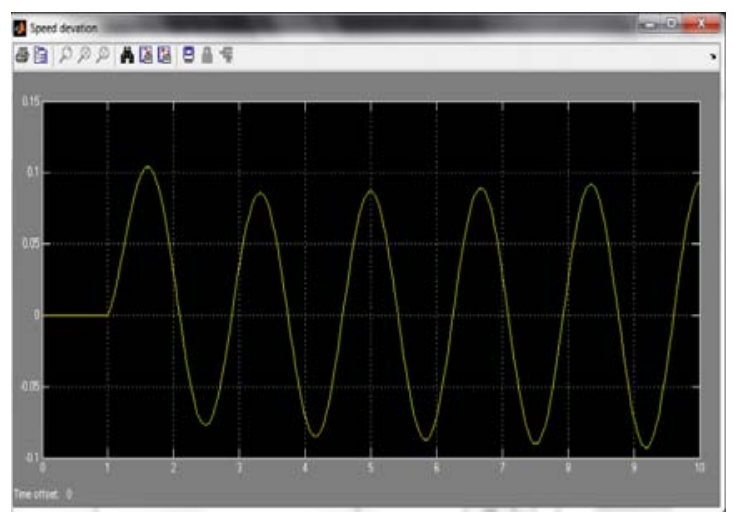

Figure 10. Speed deviation in SMIB system without UPFC

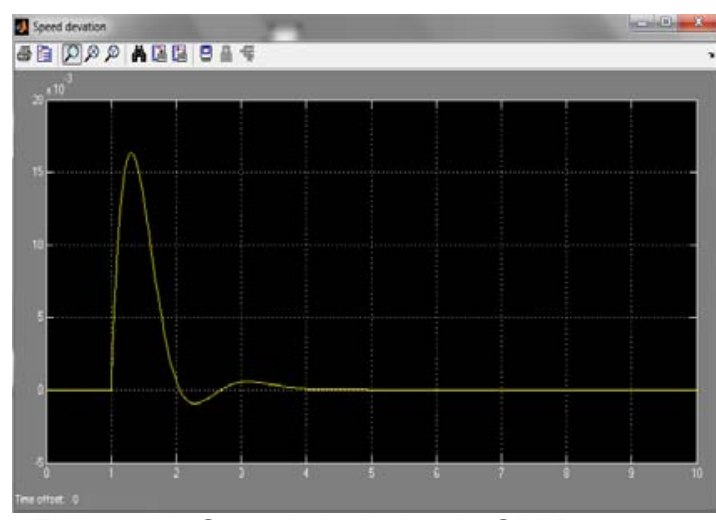

Figure 11. Speed deviation in SMIB system with UPFC 


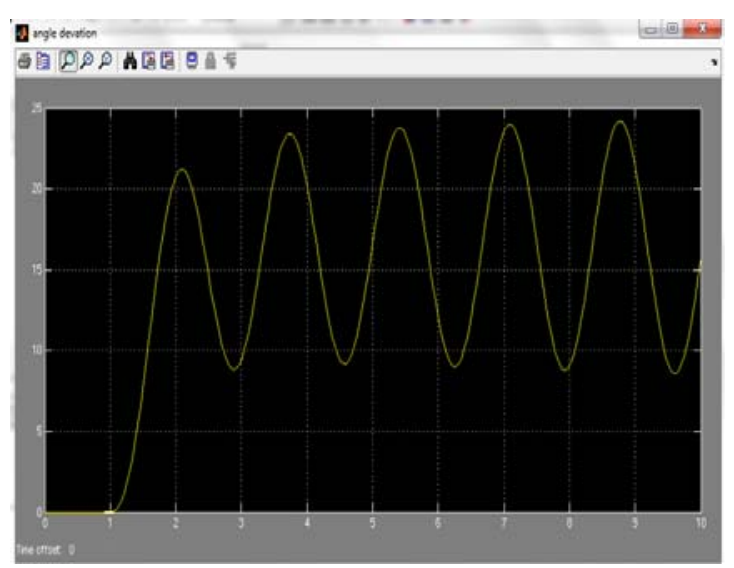

Figure 12. Angle deviation in SMIB system without UPFC

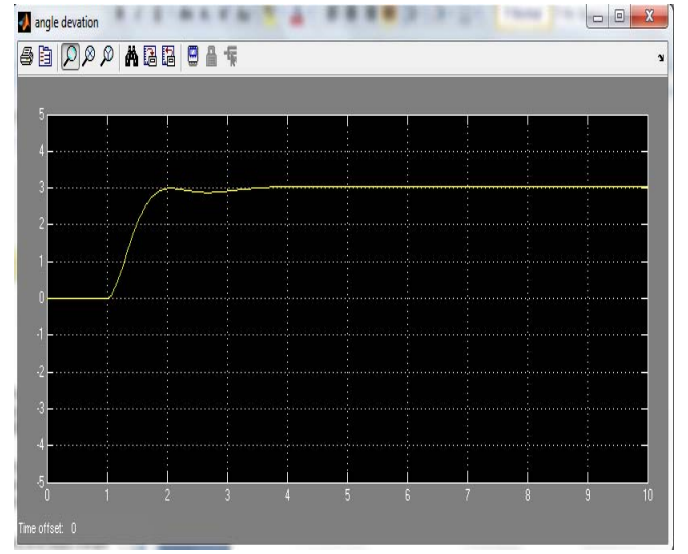

Figure 13. Angle deviation in SMIB system with UPFC

The above output comparison clearly proves that the implementation of UPFC in the SMIB system has stabilized the deviations occurring in the speed and phase angle in the system.

\section{Conclusion}

FACTS devices are improving the operation of an electric power system. The influences of such devices on steady state variables (voltage levels, transmission losses, and generating costs) are very remarkable. The benefit for each type of FACTS can be associated with its particularities and properties. They control the interrelated parameters that rule the operation of the transmission systems, including the serial impedance, the derivation impedance, the current, the voltage, the phase angle and the muffling of oscillations to different frequencies under the nominal frequency.

Today, FACTS devices are individually controlled. But according to a new EPRI report, inventive strategies incorporating system-wide control logic could further increase power transfer capability, stability and reliability of transmission systems. Controllers would be able to maximize available transfer capacity which maintaining dynamic stability and security, which could help accommodate even more electricity transactions.

The all solid-state implementation of power-flow controllers will result in a significant reduction in equipment size and installation labour, dramatic improvements in operating flexibility and performance, and a progressive reduction in capital cost that is fuelled by advances in power semiconductor technology. Furthermore, the uniform, all solid-state approach is expected to reduce manufacturing cost and lead-time by allowing the use of standard, prefabricated power inverter modules in different applications. All these will hasten the broad application of the FACTS concepts and the achievement of its ultimate goal, the higher utilization of electric power systems.

The significant contributions of the research work presented in this report are as follows:

a) A systematic approach for designing UPFC based controllers for damping power system oscillations has been presented.

b) The performance of the alternative damping controller, (ie, damping controller has been examined considering wide variation in the loading conditions and line reactance Xe.

The implementation of damping controller stabilizes the response of an alternating system to a great extent.

\section{References}

[1] Gyugyi L. Reactive power generation and control by Thyristor circuits. IEEE Trans. Ind. Appl. 1979; 5 1-15.

[2] Hingorani NG. High power electronic and flexible AC transmission system. IEEE Power Eng. Reo. 1988.

IJEEI Vol. 3, No. 3, September 2015: $141-149$ 
[3] Christ LN, et al. Advanced series compensation with variable impedance. EPRI Workshop on FACTS. Cincinnati, Ohio, USA. 1990

[4] KS Smith, L Ran, J Penman. Dynamic Modelling of a Unified Power Flow Controller. IEE ProceedingsC. $1997 ; 144(1): 7$.

[5] HF Wang. Damping Function of Unified Power Flow Controller. IEE Proceedings-C. 1999; 146(1): 81.

[6] Dr Gyugyi. Unified power-flow control concept for flexible AC transmission systems. IEE ProceedingsC. $1992 ; 139(4)$.

[7] L Gyugyi, CD Schauder, et al. The Unified Power Flow Controller: A New Approach to Power Transmission Control. IEEE Transactions on Power Delivery. 1985; 10(2): 1085.

[8] A Nabavi-Niaki, MR Iravani. Steady-state and Dynamic Models of Unified Power Flow Controller (UPFC) for Power System Studies. IEEE Transactions on Power Systems. 1996; 11(4): 1937.

[9] HF Wang. A Unified Model for the Analysis of FACTS Devices in Damping Power System Oscillations - Part III: Unified Power Flow Controller. IEEE Transactions on Power Delivery. 2000; 15(3): 978.

[10] HF Wang. Applications of Modelling UPFC into Multi-machine Power Systems. IEE Proceedings-C, 1999; 146(3): 306.

[11] N Tambey, Prof M L Kothari. Unified Power Flow Controller (UPFC) Based Damping Controllers for Damping Low Frequency Oscillations in a Power System. 2002. 